\title{
Fault Diagnosis in Flywheels: Case Study of a Reaction Wheel Dynamic System with Bearing Imperfections
}

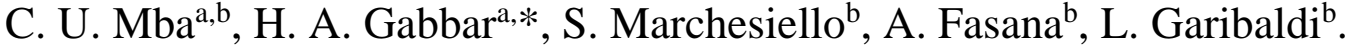 \\ ${ }^{a}$ Faculty of Engineering and Applied Science, University of Ontario Institute of Technology, Oshawa, Ontario, Canada L1H7K4. \\ ${ }^{b}$ DIRG, Department of Mechanical and Aerospace Engineering, Politecnico di Torino, 10129, Torino, Italy.
}

\begin{abstract}
This study is intended to highlight the importance of flywheels, in particular reaction wheels and steps that can be taken to ensure that they remain in stable condition for the duration of their mission. While there is an ample amount of work on this topic, this study provides an easy to follow innovative two step approach to tackle the said issue and a starting point for a different kind of analysis based on vibrations. The proposed methodology starts by obtaining the vibration data to be analyzed and applying a data based feature extraction technique known as Stochastic Resonance (SR) to the data. SR is a fairly novel tool which has shown a lot of promise in the context of mechanical systems fault diagnosis. The results obtained from the application of SR to the data is usually in the time domain but is converted to the frequency domain to reveal more information which can be used to take appropriate corrective action. As a safeguard, vibration suppression using nonlinearity which is an emerging tool is applied as a second step, to counteract whatever vibrations that may occur, thus leading to a more stable reaction wheel system.
\end{abstract}

Keywords: Reaction wheel; dynamic system; fault diagnosis; Stochastic Resonance; micro-vibration suppression; non-linearity.

(Submitted on April 9, 2017; Revised on June 12, 2017; Accepted on June 18, 2017)

(C) 2017 Totem Publisher, Inc. All rights reserved.

\section{Introduction}

Due to the ever growing threat posed to the environment as a consequence of the use of non-renewable sources of energy, there is presently plenty of ongoing research and investment in renewable energy technologies. Without a doubt, flywheels are an essential part of majority of platforms for sustainable energy. This is as a result of their high efficiency, high energy density and power, wide operating temperature range and long life cycle [1]. Flywheels work by storing kinetic energy and releasing it when it is needed. In addition, flywheels have a wide range of applications. These include but are not limited to spacecraft, vehicles and power supply. Flywheels are needed to maintain the orientation of spacecraft payloads while in motor vehicles, they sustain the crankshaft angular velocity. Some research has recently been done on the potential of flywheels to be used as a means to charge electric buses with the overall goal of reducing pollution. When it comes to electricity, flywheels have proven to be a valuable asset for constant power supply. In spite of all these advantages, safety remains a vital issue. With a flywheel rotating at very high speed, if the stability of the rotating part suddenly degenerates, there could be a large release of energy (sometimes known as an explosion) which can be quite fatal. For this reason, it is essential that proper care and various considerations are taken into account during the design of flywheel systems to ensure that they remain in optimal condition for the duration of their work.

* Corresponding author. Tel.: +19057218668 ext. 5497.

E-mail address:hossam.gabbar@uoit.ca. 
Flywheels that are used in spacecraft are known as reaction wheels and that is the area of focus in this research. A spacecraft in orbit faces a variety of harsh conditions which occasionally requires readjustment of their attitude [2]. This is where reaction wheels are crucial - they help in adjusting the attitude and pointing accuracy of spacecraft. The absence of a reaction wheel could lead to the loss of the spacecraft mission. Hence, it is vital that reaction wheels are not only present, but are also in optimal condition for the entirety of the spacecraft mission. The Reaction Wheel Assembly (RWA) is usually a mechanical one and as with all mechanical systems, vibrations do occur. These vibrations which are known as microvibrations have a low amplitude and usually occur in frequencies ranging from a few $\mathrm{Hz}$ to about a thousand $\mathrm{Hz}$ in the microgravity environment, and they could linger for a while [3].

The main source of the micro-vibrations in the RWA comes from rolling element bearings, flywheel mass imbalance, motor ripples and internal resonance [4]. Magnetic bearings have been proposed as a means to limit problems such as friction, operating temperature and lubrication which are factors that adversely affect mechanical bearings [5,6]. Magnetic bearings generally do not produce micro-vibrations and are heavier than rolling element bearings, thus they are more suited for very high speeds. However, they are expensive and quite complex to design. Moreover, losses such as iron losses, hysteresis losses, eddy current losses and air losses do occur in magnetic bearing which can pose a considerable limitation [7]. For budget reasons, rolling element bearings are still preferred to magnetic bearings in reaction wheels and different fault detection and isolation techniques have been put forward in literature as a means to enhance the overall integrity of RWAs that utilize mechanical bearings $[2,8,9,10,11]$.

\subsection{Related work}

There are different intriguing studies that have been done on detecting and isolating faults in RWAs. In [2], a clustering method known as C-Kernel Fuzzy C-Means (C-KFCM) was used via numerical simulations to identify problems in RWAs. The algorithm determines precise class centers and uses similarity class to distinguish faulty data from healthy data with a high percentage of accuracy. In this way, the limitations of the more archaic KFCM are overcome. In [8], the authors designed a fault detection and isolation module that is made up of a couple of filters. This is made possible through a combination of a nonlinear geometric and neural network approach. It is shown using numerical simulations that the approach can identify faults that affect RWA measurements. The authors in [6] took a different approach to address RWA fault issues by designing a high speed active magnetic bearing that can run in a vacuum and allow the reaction wheel size to be smaller although the total mass becomes heavier. Reference [5] proposes a Computer Aided Engineering (CAE) approach to design active magnetic bearings which requires an increase in power during operation and shows problems with flux coupling effects. Principal Component Analysis (PCA) was used for fault detection and isolation of a spacecraft reaction wheel during a particular phase of the spacecraft mission by using a simulator [10]. This requires building a PCA model from measurements of the rate of rotation of the reaction wheel. Moreover, uncertainty is not taken into account which can lead to errors in diagnosis. Reference [11] suggests a design with a focus on lubrication to ensure that mechanical bearings last for a very long time during their operation in RWAs. In [9], envelop analysis is used as a tool for identifying faults in rolling element bearings of RWAs. While all these studies are interesting, they are quite mathematically demanding. In addition, most of the methodologies used usually depend on the accuracy of the model of RWAs and make use of state space equations. Since RWA dynamics are quite complex to model, a lot of assumptions is made and thus, the methodologies fail to adequately capture a holistic scenario of RWA output. One of the objectives in this paper is to apply SR which is a simple dynamic nonlinear equation to the output from RWAs or their bearings. SR is a vibration data based technique and as a result, it does not need a mathematical model of the system that is to be analyzed. It makes use of noise to its advantage and as a consequence, there is no need to filter the data. Furthermore, it is very easy to apply and it is not as mathematically demanding as any of the procedures mentioned in this review. The computational time of SR is quite reasonable and the results obtained in the frequency domain can be used to take immediate corrective actions if required. At the end, it is intended to introduce SR as a suitable tool for micro-vibration based condition monitoring in RWAs during the initial test phase. The second objective which involves applying vibration suppression to RWA is a safeguard that is used to make sure that micro-vibrations that do occur do not cause any problems. The use of nonlinearity for vibration suppression is an emerging tool and it is used in this paper. Like SR, it is quite simple to apply and works well over a range of frequencies. Overall, this paper integrates these two approaches in order to achieve the goal of preventing failures in RWAs and this is shown in an example of an RWA with faulty bearings. 


\subsection{Methodology}

The scope of this research is twofold and it is the beginning of a different kind of study that will be carried on to real life scenarios. First, using numerical simulations, rolling element bearing imperfections from an RWA is used as a case study to show how effective an innovative feature extraction data based technique known as Stochastic Resonance (SR) can be when used in the micro-vibration test that is usually performed on RWAs during the initial phase. SR is a time domain technique but its application also extends to the frequency domain which makes it a very suitable tool for this case study. Second, a vibration suppression procedure using non-linearity is applied to a broad model (both linear and nonlinear) that can be representative of a simple balanced RWA with disturbances from the faulty bearings. This second step is a precaution that is used in spacecraft to ensure that micro-vibrations that may occur are contained. The combination of these two approaches provides a novel systematic method and platform for giving the mechanical integrity of RWAs a boost. The flowchart in figure 1 demonstrates the methodology and scope of this work.

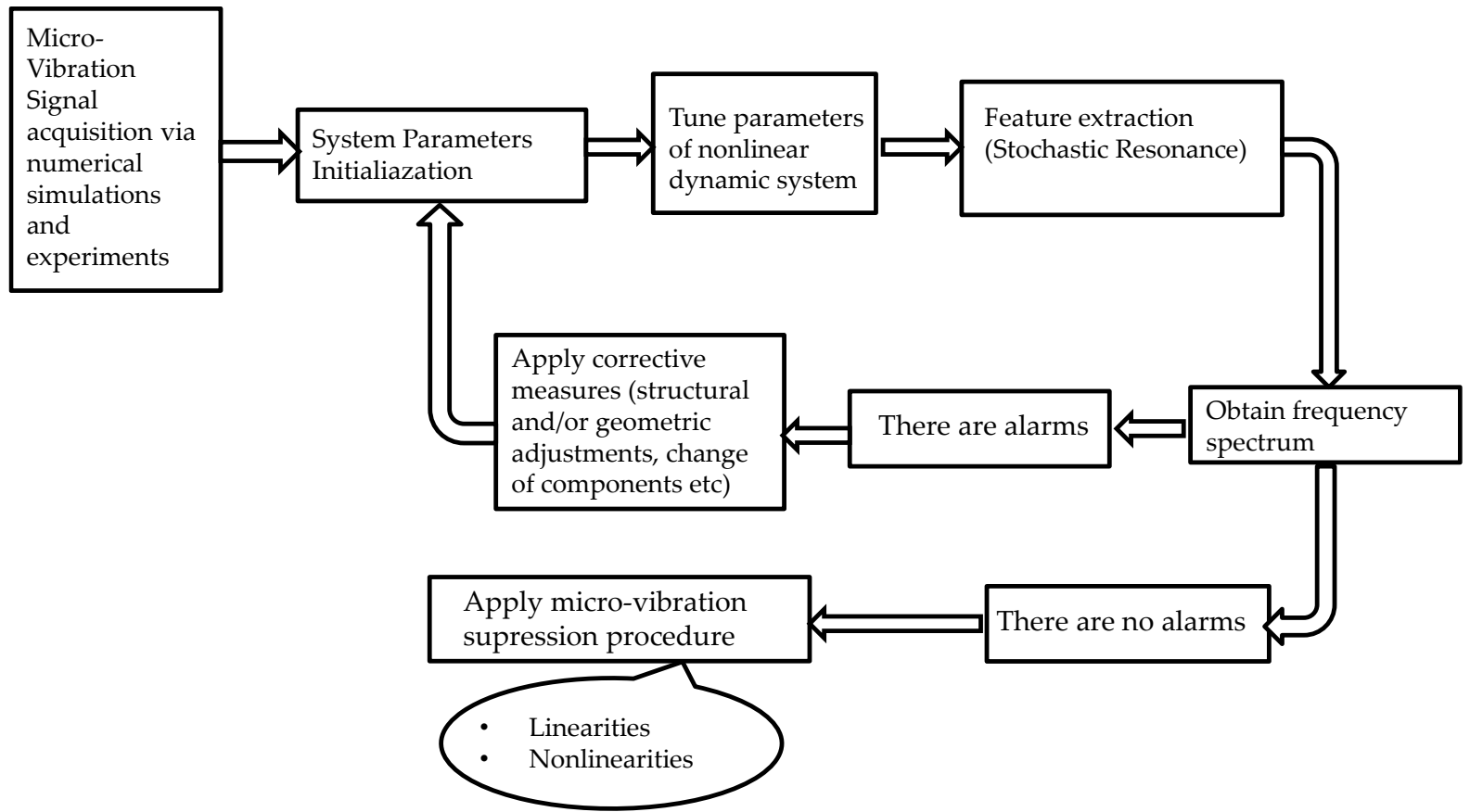

Figure 1. Flowchart illustrating the proposed methodology as part of the platform for enhancing the integrity of RWAs

\section{Stochastic Resonance}

SR is a non-linear time domain approach that was first used within the framework of the earth's climate [12]; however its application has been extended to other fields such as physics, chemistry, finance, biology and engineering [13]. Going by its broad definition, SR describes a situation where the existence of noise in a non-linear system is used to strengthen the system's output in terms of its Signal to Noise Ratio (SNR). In other words, rather than degradation in system performance because of noise, SR works with noise and uses noise to its advantage. It is governed by the non-linear dynamic equation:

$$
\frac{d x}{d t}=-\frac{d U(x)}{d x}+s(t)+n(t)
$$

Where $n(t)$ is the noise that is either inherently present in the system or synthetically introduced to the system, $s(t)$ is the input signal which is periodic and $U(x)$ is a term known as the potential function and it is given by:

$$
U(x)=-\frac{1}{2} a x^{2}+\frac{1}{4} b x^{4}
$$

$U(x)$ contains parameters $a$ and $b$ which have to be masterfully tuned in order to obtain desired results. Substituting (2) into (1) gives the SR equation:

$$
\frac{d x}{d t}=a x-b x^{3}+s(t)+n(t)
$$


In order to illustrate how this phenomenon works, let's consider the Bistable system shown in figure 2.

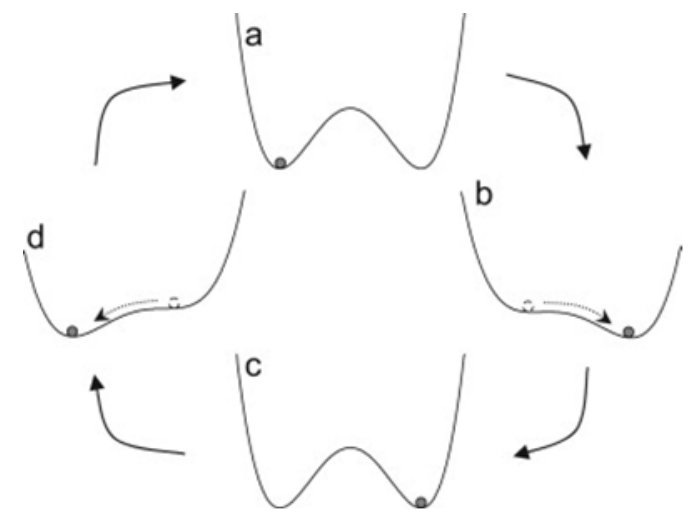

Figure 2. Bistable system showing Brownian particle motion in the presence of a periodic input signal and noise [14]

The figure shows a double well symmetric potential with four different states a, b, c and d. There is a ball known as a Brownian particle in one of the wells of each potential. When a periodic input signal is applied to the potential in a, both the left side and the right side wells modulate but this modulation does not cause the Brownian ball to move to the next well. When a certain amount of noise is properly combined with the periodic input signal and applied to the potential, the addition of noise gives the Brownian particle enough energy to move between wells. This is what happens in $b$, $c$ and d. In the absence of both the periodic input signal and noise, the behavior of the potential depends on initial conditions [15]. Assuming that a in figure 2 is the original state of a non-linear system, the parameters a and $b$ in equation 3 can be tuned in such a way that the Brownian particle jumps to the height of the barrier between the wells without getting into the right side well but rather returning to left side well. This event produces a spike that can be quantified by a performance indicator such as kurtosis and can be used to detect tiny or hidden impulses in a noisy signal. Figure 3 demonstrates such a scenario.
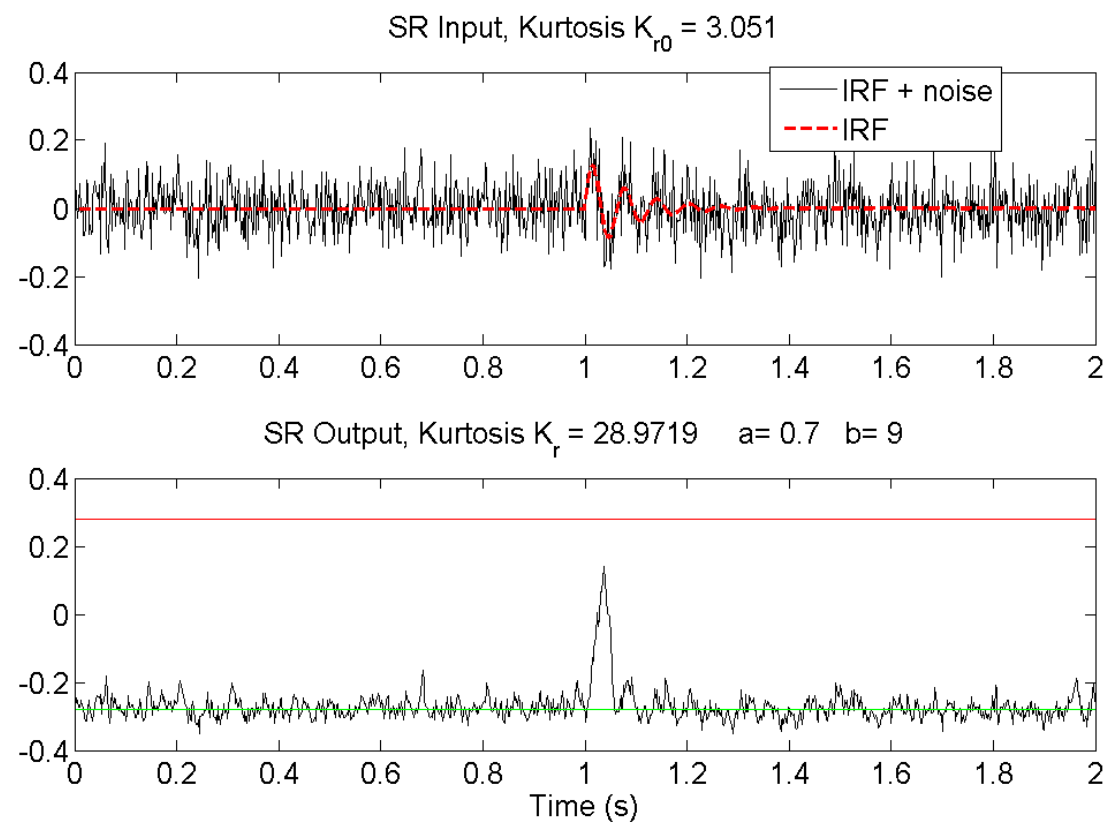

Figure 3. Example showing results that can be obtained when a and b are well-tuned [14]. Note that in the bottom figure, the green horizontal line represents the left side of the well and the red horizontal line represents the right side of the well.

When an Impulse Response Function (IRF) submerged in noise is applied to the potential, the parameters a and $b$ are tuned in such a way that the SR output gets to the peak of the potential barrier and almost gets into the right side well but does not, instead it returns to the left side well. This results in a clear amplification of the impulse as can be seen in the 
bottom of figure 3 . The green horizontal line represents the left side of the well and the red horizontal line represents the right side of the well. The vertical distance from either of the lines to zero represents the height of the barrier.

Equation 3 holds true for systems with very small amplitude, frequency and noise intensity but realistic systems do not meet the requirements of very small amplitude, frequency and noise intensity. For equation 3 to work properly when used on a system, pre-processing techniques like scale normalization, modulation, rescaling frequency, etc. are applied to the system before equation 3 is applied. Like the work in [16], re-scaling frequency which is an uncomplicated technique is used here. Furthermore, queries come up when it comes to the issue of how best to tune the parameters a and b. Although there are researches both present and ongoing that show how best to go about this matter, what has been shown to be quite effective and simple to apply is normalizing the input signal with a standard deviation of 0.07 before defining a fixed range for a $\in$ [.1,1] with an interval of 0.1 and $b \in[1,11]$ with an interval of 1 . Then a search is performed within the defined ranges to find the maximum kurtosis. Finally, the combination of $a$ and $b$ that gives the maximum kurtosis within the defined range is selected and used to find the corresponding SR output.

\subsection{Simulated Micro-Vibration Data}

As mentioned in the introduction, micro-vibration tests are carried out on RWAs at the initial test phase. This is performed on a Kistler table [9]. In [9], rolling element bearing imperfections are simulated as close as possible to real RWA bearings. Faulty mechanical bearings emit distinct impulses when the rolling element moves over the fault. The period of the generated impulse train has a well-defined relationship with the bearing characteristic frequencies which are associated with components (outer race, inner race, cage and rolling element) of the bearing and can be used to identify the faulty component.

In this paper, the micro-vibration from an RWA bearing is simulated based on the simulations in [9]. Since the outer race rotates and the inner race is fixed in reaction wheel applications, we only consider faults in the outer race and rolling element. That is to say that two different fault cases are assessed - outer race and rolling element faults. The simulated micro-vibration data is sampled at $10000 \mathrm{~Hz}$ because high sampling frequencies are not obtainable in micro-vibration tests. The base plate resonance frequency is set at $450 \mathrm{~Hz}$ while the shaft speed is $25 \mathrm{~Hz}$. The signal is buried in Gaussian noise with an intensity of 0.05 and a standard deviation of 0.07 . Figures 4 and 5 show the results obtained when SR is applied to the simulated RWA bearing data with rolling element and outer race faults.

As can be seen in figure 4, the effect of SR is very obvious. Before the application of SR, the defect frequency corresponding to the rolling element (BSF) is non-existent. On the application of SR, the hidden frequency is amplified thus making it detectable. Generally, rolling element faults are modulated at the cage speed (FTF) so the harmonics of the rolling element defect frequency will have sidebands that are spaced at the cage speed on both sides. In figure 5, the effect of SR can be seen after its application to the simulated data. Before SR is applied, the frequency that matches the outer race is not visible. Nevertheless, SR makes the frequency quite discernible. On the basis of these results, changes or modifications can be made accordingly in an RWA.

We note here again that mechanical bearing imperfections have been used as an example as there can be other sources of micro-vibration in an RWA such as flywheel mass imbalance, motor ripples and internal resonance [4]. The aim of this illustration is to demonstrate how potent SR can be if used during the micro-vibration initial test. In addition to standing out in this regard and also being suitable for micro-vibration tests, SR magnifies impulses that are hidden in noise and can reveal information that might be useful for making structural or geometric readjustments. Compared to other methods found in literature for detecting faults in reaction wheels, SR is very straightforward and not mathematically demanding.

\section{Micro-Vibration Suppression}

After the initial micro-vibration test, measures such as changes in geometry design, components, operating temperature and speed, are taken to ensure that micro-vibrations do not occur in the RWA. Nonetheless due to harsh and unpredictable conditions in orbit, micro-vibrations may still occur. That is why RWAs are fitted with micro-vibration isolation systems as a safeguard to ensure that micro-vibrations that may occur do not interrupt the spacecraft mission. 

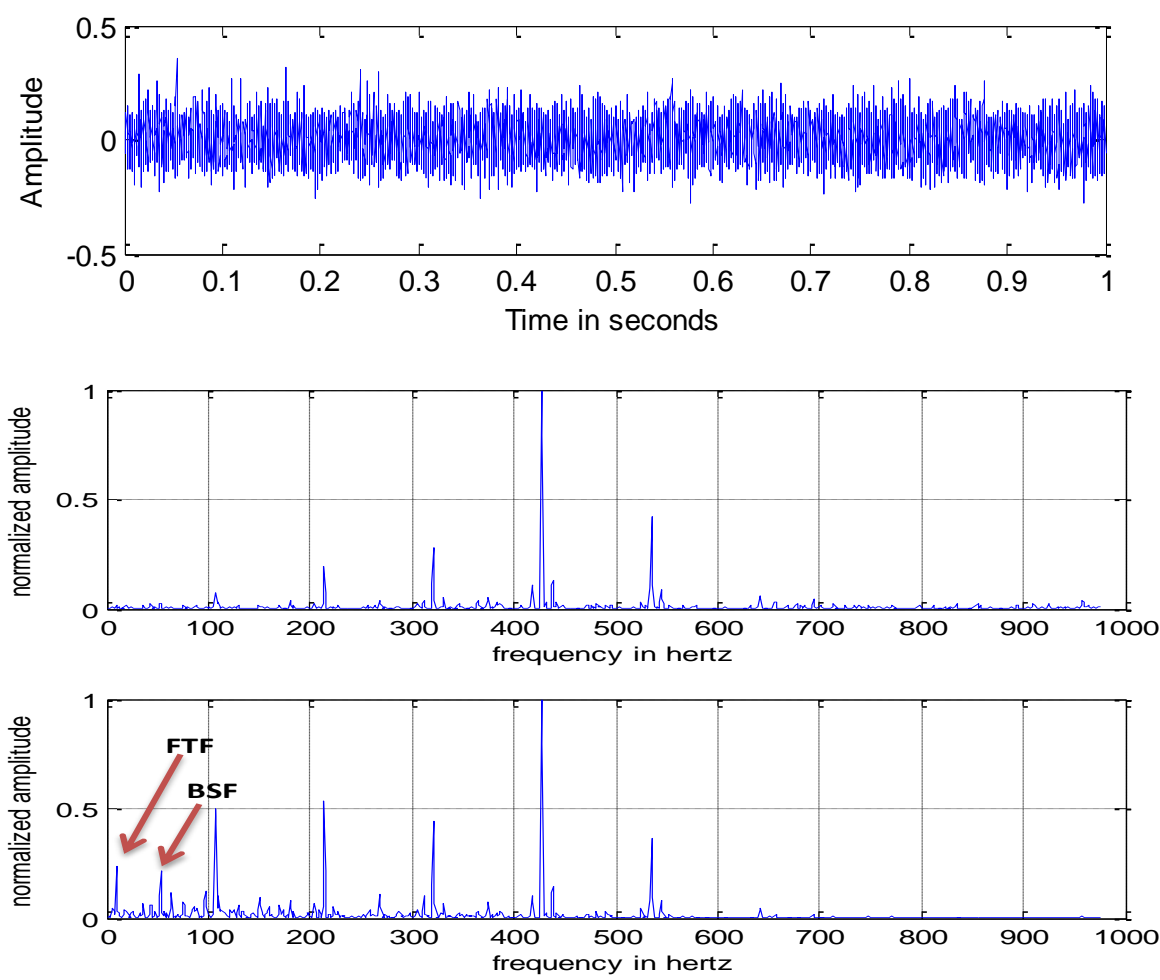

Figure 4. Plots showing simulated RWA bearing data in the time domain and the results before and after SR in the frequency domain. The middle plot is before SR and bottom plot is after SR. Fundamental Train Frequency $(\mathrm{FTF})=9.7222 \mathrm{~Hz}$, Ball Spin Frequency $(\mathrm{BSF})=53.4722 \mathrm{~Hz}$
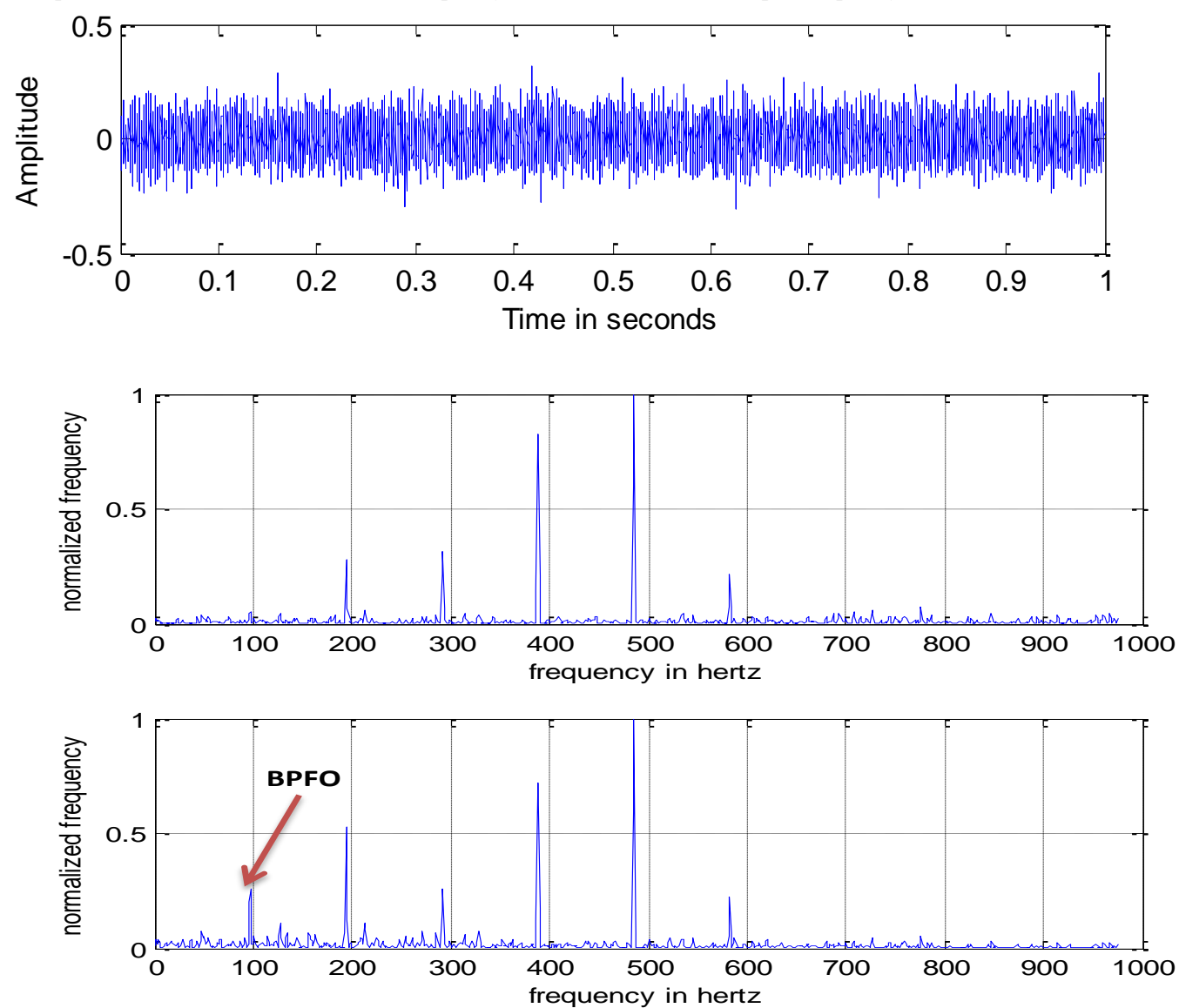

Figure 5. Plots showing simulated RWA bearing data in the time domain and the results before and after SR in the frequency domain. The middle plot is before SR and bottom plot is after SR. Ballpass Frequency Outer Race $($ BPFO $)=97.2222$ 
According to [3], there are four main ways for micro-vibration isolation. These include active, passive, active-passive hybrid and semi-active. The active isolation technique is quite effective for low frequency amplitudes and makes use of sensors and actuators to regulate the system parameters. However, it requires external power. The passive isolation technique does not require external power and it is very effective for dampening out high frequency amplitudes. Its disadvantage lies in the fact that it is not useful when there are low frequency amplitudes. So the active-passive hybrid technique tries to avoid the downside of a pure passive or pure active isolation technique by combining their benefits. Still, this carries the same con as a pure active isolation procedure. The semi-active approach offers more reliability than the pure active or active-passive hybrid approach even if the active part fails. However, it is rarely used in spacecraft due to weight constraints.

Recently new techniques that make use of non-linearity have been explored. As stated in [3], these techniques offer good performance and show great promise. Moreover, they can be executed with passive modules which offer the cheapest alternative in the suppression of micro-vibrations and work well over a wide frequency range. This is corroborated in [17] where non-linearity is used to contain certain frequency amplitudes and the results are compared with what happens when linearity is used instead. The methodology is employed here to suppress micro-vibration as follows:

For a given dynamic system using a linear system as a first case study,

$$
\begin{aligned}
& \dot{x}=A x+B U \\
& z=C x+D U
\end{aligned}
$$

where $x$ is the system state, $U$ is the control input, $z$ is the system output and $A, B, C, D$ are suitable matrixes. Given a defined frequency range $I(\omega)$ and a desired frequency output amplitude level $\tilde{z}$, find a state feedback control law $U$ that is a non-linear function of either one or some of the system states such that the output frequency amplitude is either less or equal to the desired frequency output amplitude level. That is

$$
u=f(\dot{x}, \ddot{x}, \dddot{x}, \ldots)
$$

such that

$$
\max _{\omega \in I(\omega)}(z(j \omega) z(j \omega)) \leq \tilde{z}
$$

$f$ is a non-linear function. Now let us consider a linear system that can be representative of the approximate behaviour of a simple balanced RWA with disturbances from rolling element bearing imperfections as input and an output that gives the resulting micro-vibration caused by the rolling element bearing imperfections.

$$
\begin{aligned}
& M \ddot{x}=-k_{1} x-c_{1} \dot{x}+u+\delta \\
& z=k_{1} x+c_{1} \dot{x}-u
\end{aligned}
$$

where $M$ is the mass of the system, $k_{1}$ is the stiffness, $c_{1}$ is the damping, $u$ is the control input, $\delta$ is the bearing microvibration disturbance and $z$ is the system output. The numerical values of these parameters are selected as $M=5.6204 \mathrm{Kg}$, $k_{1}=1,600 \mathrm{KN} / \mathrm{m}$ and $c_{1}=1 \mathrm{Ns} / \mathrm{m}$. The control input $u$ here is selected as $-c_{2} \dot{x}$ for the linear case and through a bit of trial and error as $-c_{2} \dot{x}^{1 / 2}$ for the non-linear case where the gain $c_{2}=10$. It was found for this particular case that other nonlinear control inputs like $-c_{2} \dot{x}^{2},-c_{2} \dot{x}^{3},-c_{2} \dot{x}^{4} \ldots c_{2} \dot{x}^{12}$ gave the same results and no improvement when compared to the results obtained in the absence of a control input. Thus only the results obtained when the non-linear control input is $-c_{2} \dot{x}^{1 / 2}$ are shown here. Figures 6 and 7 show the results obtained when there is no control input and when there is a linear and non-linear control input.

As can be seen on the left hand side of figure 6, the disturbance signal has smaller maximum amplitude when compared to the amplitude of the RWA output on the right hand side. This suggests that a smaller micro-vibration can induce larger micro-vibrations as the vibrations propagate through the system structure. Also, the presence of very tiny or no environmental damping in orbit can make this sort of situation worse [3]. The right hand side of figure 6 shows the results obtained in the absence of any control input while the top of figure 7 shows the results obtained when the linear control input $u=-c_{2} \dot{x}$ is applied to the RWA dynamic system. It can be seen from both figures that the maximum amplitude is suppressed by a good amount in the presence of the linear control input. The suppression is increased considerably (by $91 \%$ when compared to the maximum amplitude of the original signal) when the non-linear control input is introduced as can be seen on the right hand side of figure 7 . In fact, the maximum amplitude becomes smaller than that of the disturbance on the 
left hand side of figure 6. It has been shown in literature that an increase in gain of the linear control input gives better results around the system fundamental frequency at the cost of performance deterioration at higher frequencies [17]. That means when a linear control input is used, great care should be taken in tuning the gain of the linear control input. Apparently this problem is overcome when some non-linearity is introduced.
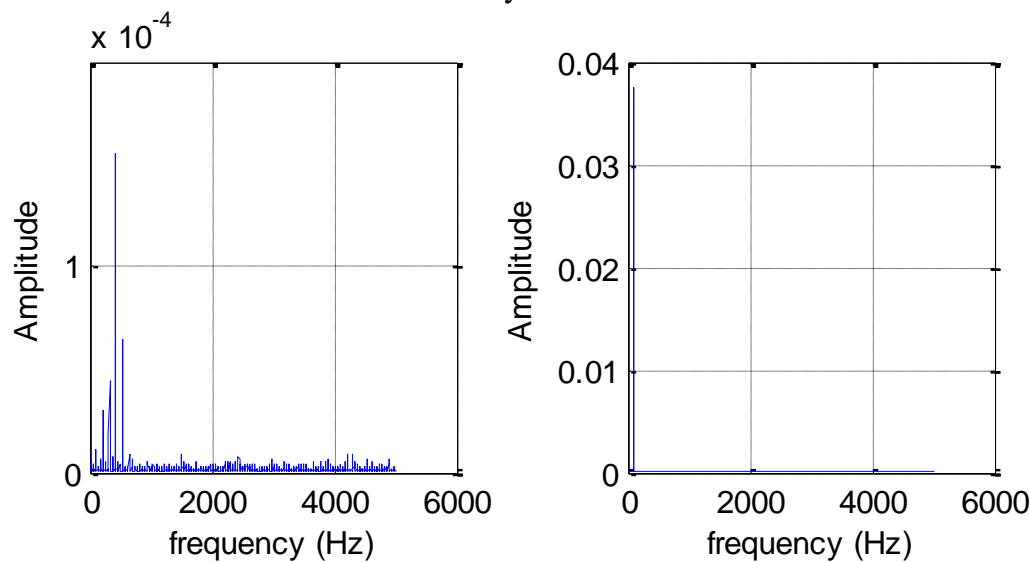

Figure 6. Spectra showing input and output of the RWA. Left hand side shows the input disturbance from the bearing with imperfections in the rolling element and right hand side shows output of the RWA without control input.
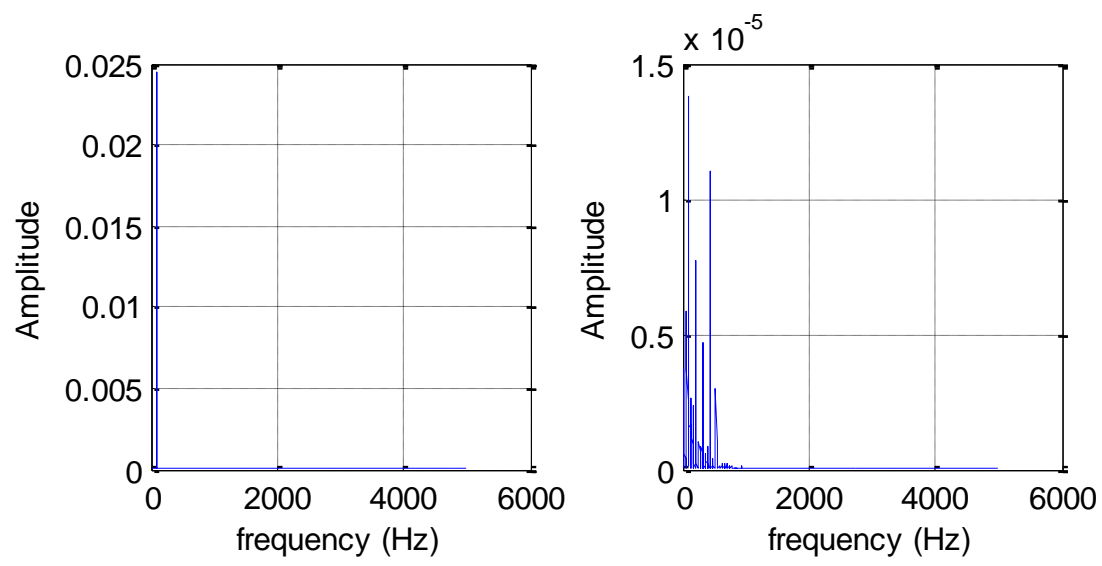

Figure 7. Spectra showing output of the RWA with input disturbance from bearing with rolling element fault. Left hand side shows the output in the presence of a linear control input $u=-c_{2} \dot{x}$ and right hand side shows the output in the presence of a non-linear control input $u=-c_{2} \dot{x}^{1 / 2}$.
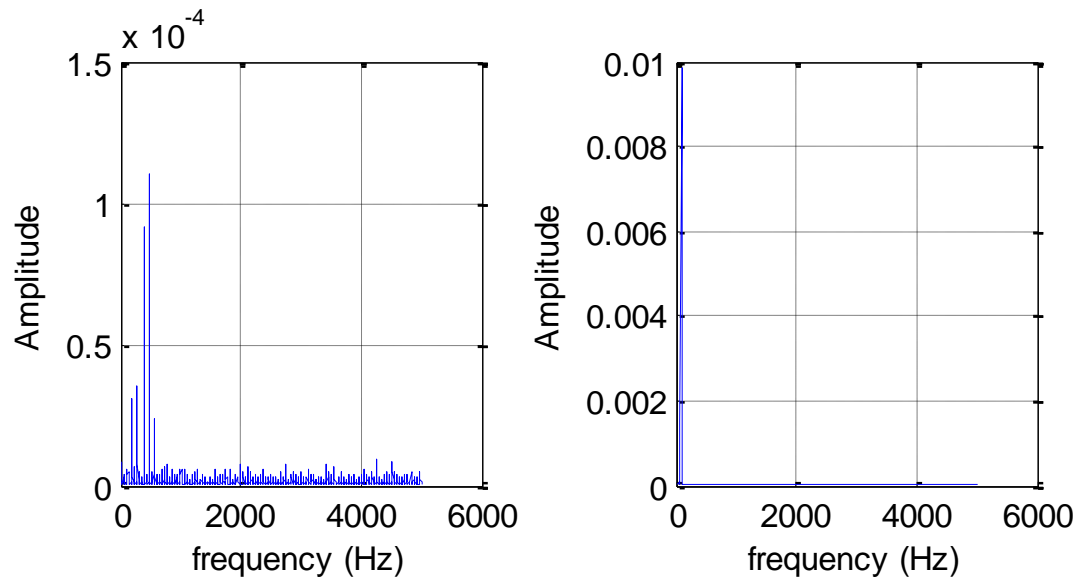

Figure 8. Spectra showing input and output of the RWA. Left hand side shows the input disturbance from the bearing with imperfections in the outer race and right hand side shows output of the RWA without control input. 

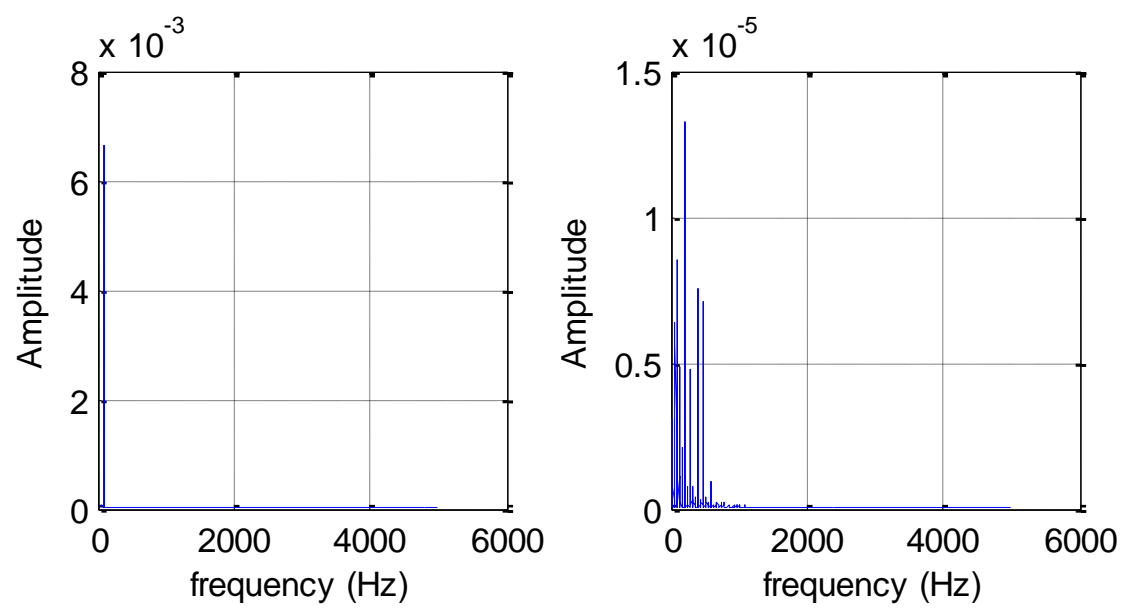

Figure 9. Spectra showing output of the RWA with input disturbance from bearing with outer race fault. Left hand side shows the output in the presence of a linear control input $u=-c_{2} \dot{x}$ and right hand side shows the output in the presence of a non-linear control input $u=-c_{2} \dot{x}^{1 / 2}$.

When the disturbance from the bearing with outer race fault is used as the input disturbance of the RWA dynamic system, results similar to the ones in figures 6 and 7 are obtained as shown in figures 8 and 9. The micro-vibration on the left hand side of figure 8 induces micro-vibrations with larger amplitude as can be seen on the right hand side of the same figure. In this case, there is no control input. The linear control input is introduced on the left hand side of figure 9 with the result being a good suppression of the micro-vibration maximum amplitude and when the non-linear control input is introduced on the right hand side of the same figure, there is a further suppression (by $88 \%$ when compared with the maximum amplitude of the original signal) of the maximum amplitude of the micro-vibration maximum amplitude which goes below the original maximum amplitude of the faulty bearing.

While it is reasonable to first test out new approaches on very simple linear cases via simulations, in reality, the behaviour of dynamic systems are very rarely linear and as a consequence, it is always logical to extend the test of new approaches to non-linear system simulations. In order to test out the effectiveness of using non-linearity for vibration suppression in a non-linear system, non-linearity is intentionally introduced in (7a) and (7b) to obtain (8a) and (8b) as follows:

$$
\begin{aligned}
& M \ddot{x}=-k_{1} x-c_{1} \dot{x}-\xi x^{3}+u+\delta \\
& z=k_{1} x+c_{1} \dot{x}+\xi x^{3}-u
\end{aligned}
$$

where $\xi x^{3}$ is a non-linearity and $\xi$ must be carefully chosen in such a way that the dynamic system behaves in a nonlinear way. As a measure to ensure this, a plot of $\boldsymbol{k}_{1} \boldsymbol{x}+\xi \boldsymbol{x}^{3}$ against $\boldsymbol{x}$ can be used to verify that the output of the dynamic system is non-linear. Figure 10 shows two cases where $\xi$ is both properly and not properly selected. The figures are generated in the absence of any control input. It can be seen from figure 10 that the system behaves in a linear manner when $\xi$ is not well selected and it behaves in a non-linear manner when $\xi$ is well selected. For the second case study which should be a non-linear dynamic system, $\xi$ is selected as $1,600 \mathrm{KN} / \mathrm{m}$ while the other parameters are the same as the linear dynamic system. Figures 11 and 12 show what happens when the non-linear dynamic system has a bearing with faults in the rolling element in the presence of no control input, a linear control input and a non-linear control input.

The input on the left hand side of figure 11 is the same as the linear case in figure 7 . On the right hand of figure 11 , it can be seen that the bearing with faults in the rolling element results to a frequency with larger amplitude in the non-linear system in the absence of a controller when compared to the linear case. In figure 12 on the left side, the linear controller does quite well to suppress the amplitude of the frequency while the non-linear controller on the right side does a better job by suppressing the maximum amplitude by $57 \%$ when compared to the maximum amplitude in the original signal. In this particular case, it was found through several trials that the non-linear controller $-c_{2} \dot{x}^{3}$ was the most effective. The results are mostly the same when the input disturbance is from the bearing with outer race faults. Figure 13 shows the input disturbance of the non-linear dynamic system which is the same as the linear case in figure 8 . Just like the case of the bearings with faults in the rolling element, there is a frequency with much higher amplitude on the right side of figure 13 which is suppressed on the left side of figure 14 by the linear control input and further suppressed (by $40 \%$ when compared with the maximum amplitude in the original signal) on the right side of the same figure by the non-linear control input. This goes on to show how effective non-linearities can be for the suppression of micro-vibration. 

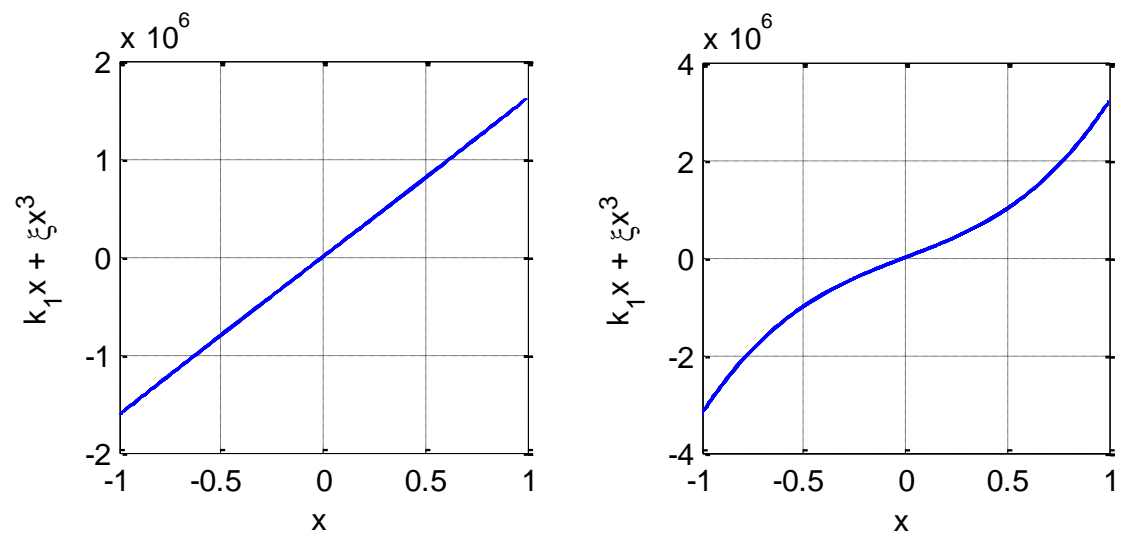

Figure 10. Plot of $k_{1} x+\xi x^{3}$ against $x$. The left side shows linearity when $\xi$ (in this case $16 \mathrm{KN} / \mathrm{m}$ ) is not properly selected and the right side shows nonlinearity when $\xi$ (in this case $1,600 \mathrm{KN} / \mathrm{m}$ ) is properly selected.
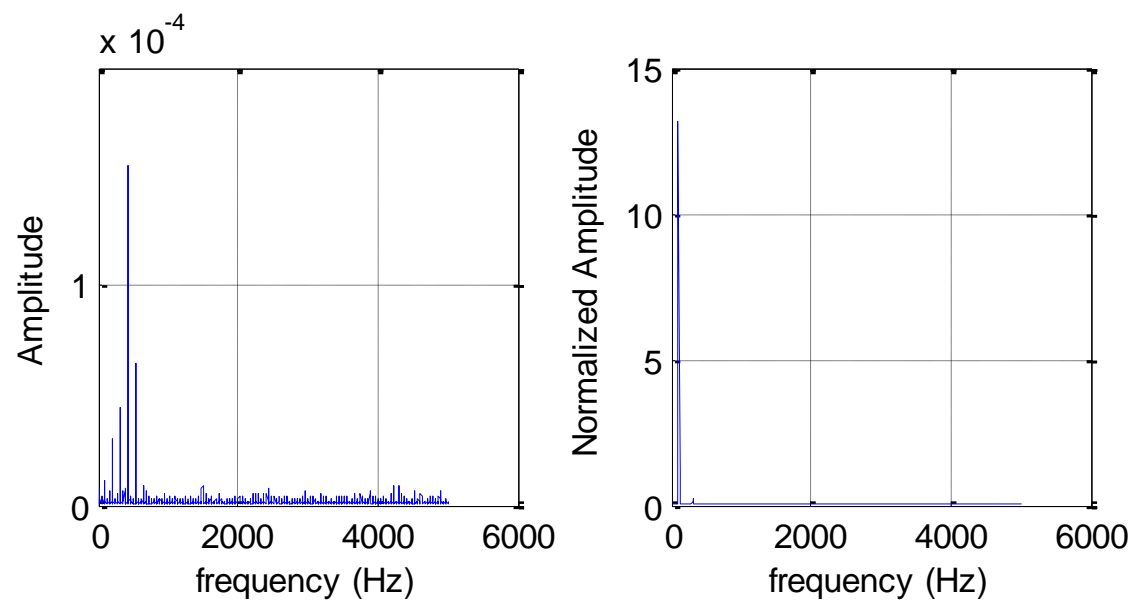

Figure 11. Spectra showing input and output of the non-linear dynamic system. Left hand side shows the input disturbance from the bearing with imperfections in the rolling element and right hand side shows output of the non-linear dynamic system without control input.
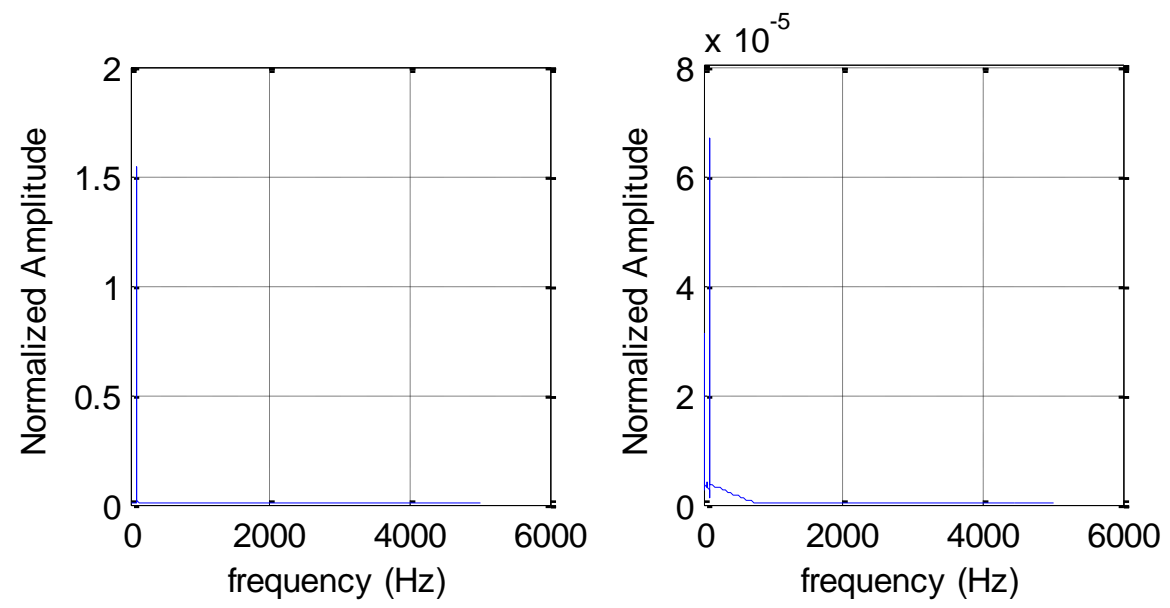

Figure 12. Spectra showing output of the non-linear dynamic system with input disturbance from bearing with rolling element fault. Left hand side shows the output in the presence of a linear control input $u=-c_{2} \dot{x}$ and right hand side shows the output in the presence of a non-linear control input $u=-c_{2} \dot{x}^{3}$. 

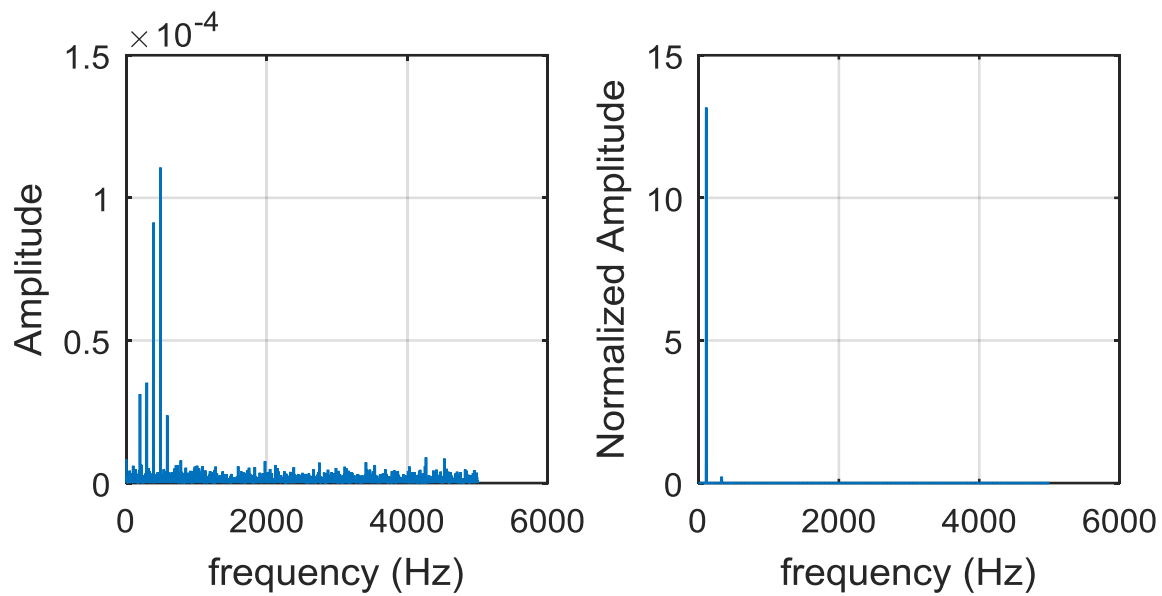

Figure 13. Spectra showing input and output of the non-linear dynamic system. Left hand side shows the input disturbance from the bearing with imperfections in the outer race and right hand side shows output of the non-linear dynamic system without control input.
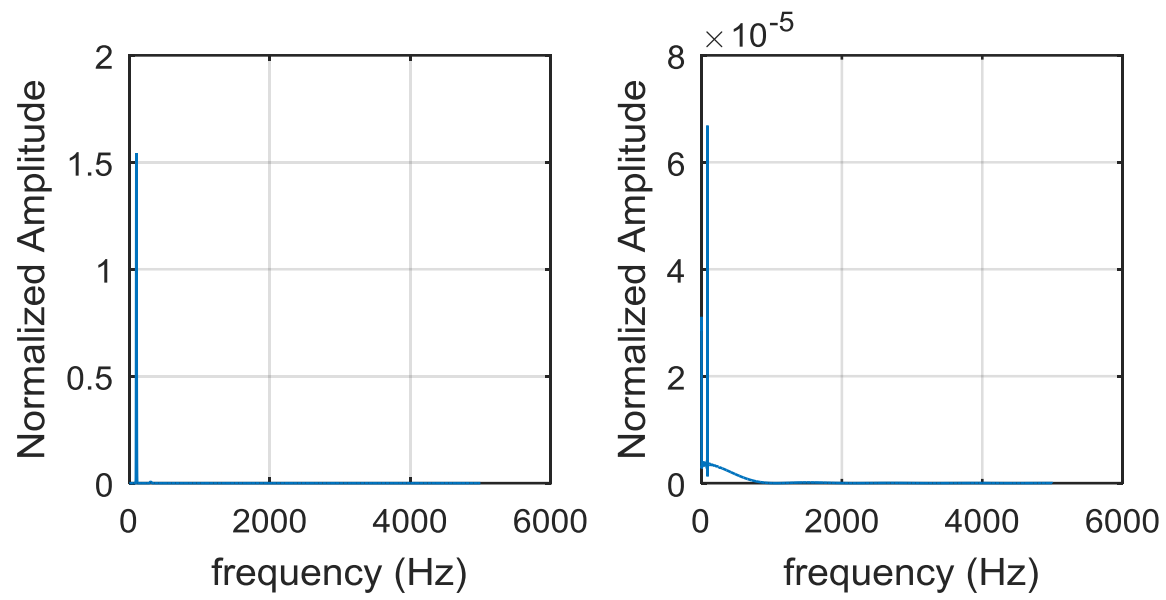

Figure 14. Spectra showing output of the non-linear dynamic system with input disturbance from bearing with outer race fault. Left hand side shows the output in the presence of a linear control input $u=-c_{2} \dot{x}$ and right hand side shows the output in the presence of a non-linear control input $u=-c_{2} \dot{x}^{3}$.

\section{Conclusions}

This paper presents a novel feature extraction approach for RWA bearings initial test phase. The procedure is a data driven time domain technique known as Stochastic Resonance (SR) which is able to harness the noise that is either intrinsic in a system or artificially introduced into the system. It works by significantly maximizing the weak or hidden impulses buried in noise when the parameters of the SR non-linear dynamic system are skillfully tuned; and it is this characteristic that is exploited in this paper. The results obtained originally in the time domain is converted to the frequency domain to reveal more information on faulty components in addition to the presence and severity of the fault. The approach offers considerable advantage over other procedures used for the same purpose in terms of reasonable complexity, computational time, use of data rather than a model and a high degree of amplification of concealed impulses. As a safeguard, microvibration suppression based on non-linearity is integrated with SR and applied in a worst case scenario of the RWA. The results indicate that the percentage of achieved vibration suppression can be as high as $91 \%$. The combination of SR and non-linear micro-vibration suppression provides a new integrated platform for detecting and isolating faults thus allowing for a substantial enhancement in the mechanical integrity of reaction wheels. 


\section{References}

1. L. Haichang, and J. Jiang, "Flywheel energy storage - An upswing technology for energy sustainability," Energy and buildings, vol. 39, no.5, pp. 599-604, May 2007

2. H. Di, A. Sarosh, and Y.-F. Dong, "A novel KFCM based fault diagnosis method for unknown faults in satellite reaction wheels," ISA transactions, vol. 51, no.2, pp. 309-316, March 2012

3. L. Chunchuan, X. Jing, S. Daley, and F. Li, "Recent advances in micro-vibration isolation," Mechanical Systems and Signal Processing, vol. 56, pp. 55-80, May 2015

4. L. Qing, D. Li, W. Zhou, J. Jiang, G. Yang, and X. Wei, "Dynamic modelling and observation of micro-vibrations generated by a Single Gimbal Control Moment Gyro," Journal of Sound and Vibration, vol. 332, no.19, pp. 4496-4516, September 2016

5. S. Michael, T. Roschke, E. Bindl, and D. Blonski, "Design and development of a compact magnetic bearing momentum wheel for micro and small satellites," in 15th Annual/USU Conference on Small Satellites, pp. 1-9, 2001

6. Z. Christof, T. Baumgartner, and J.W. Kolar, "High-speed magnetically levitated reaction wheel demonstrator," in Power Electronics Conference (IPEC-Hiroshima 2014-ECCE-ASIA) International, pp.1707-1714, May 2014

7. B. Hannes, M. Cole, P. Keogh, R. Larsonneur, E. Maslen, Y. Okada, G. Schweitzer, and A. Traxler, "Magnetic bearings: theory, design, and application to rotating machinery," Springer Science \& Business Media, 2009

8. B. Pietro, M. Blanke, P. Castaldi, N. Mimmo, and S. Simani, "Combined Geometric and Neural Network Approach to Generic Fault Diagnosis in Satellite Reaction Wheels," Ifac-papersonline, pp. 194-199, January 2015

9. M.P. Le, E. van der Heide, R. Seiler, and E.J.E. Cottaar. "Detection and diagnosis of ball bearing imperfections in reaction wheels by micro-vibration test," in 12th European Conference on Spacecraft Structures, Materials and Environmental Testing, pp.53-58, July 2012.

10. N. Othman, I. Gueddi, P. Dague, and K. Benothman, "Spacecraft actuator diagnosis with principal component analysis: application to the rendez-vous phase of the mars sample return mission," Journal of Control Science and Engineering, pp. 2232, January 2015

11. K. Sathyan, H.Y.Hsu, S.H.Lee, and K.Gopinath, "A Bearing Cartridge Assembly for Long-Term Performance of Momentum/Reaction Wheels used in Spacecraft," Tribology in Industry, vol. 36, no. 1, pp. 22-32, March 2014

12. B. Roberto, A. Sutera, and A. Vulpiani, "The mechanism of stochastic resonance," Journal of Physics A: mathematical and general, vol. 14, no.11, pp. L453-L457, November 1981

13. M.D. McDonnell, and D. Abbott, "What is stochastic resonance? Definitions, misconceptions, debates, and its relevance to biology," PLoS Comput Biol, vol. 5, no.5, pp. 1-9, May 2009

14. S. Marchesiello, A. Fasana, and L. Garibaldi, "Best parameter choice of Stochastic Resonance to enhance fault signature in bearings," in International Conference on Structural Engineering Dynamics, pp. 1-7, June 2015

15. G. Luca, P. Hänggi, P. Jung, and F. Marchesoni, "Stochastic resonance," Reviews of modern physics, vol. 70, no.1, pp. 223-287, January 1998

16. C.U. Mba, S. Marchesiello, A. Fasana, and L. Garibaldi, "Vibration Based Condition Monitoring of Spur Gears in Mesh using Stochastic Resonance," in Surveillance 8 International Conference, pp. 1-15, October 2015

17. X.J. Jing, Z.Q. Lang, S.A. Billings, G.R. Tomlinson, "Frequency domain analysis for suppression of output vibration from periodic disturbance using nonlinearities," Journal of Sound and vibration, vol. 314, no.3, pp. 536-557, July 2008 